\title{
Chaotic bound state of localized structures in the complex Ginzburg-Landau equation
}

\author{
Dmitry Turaev, ${ }^{1, *}$ Andrei G. Vladimirov, ${ }^{2, \dagger}$ and Sergey Zelik ${ }^{3, \dagger}$ \\ ${ }^{1}$ Ben Gurion University, P.O.B. 653, 84105 Be'er Sheva, Israel \\ ${ }^{2}$ Weierstrass Institute for Applied Analysis and Stochastics, Mohrenstrasse 39, D - 10117 Berlin, Germany \\ ${ }^{3}$ Department of Mathematics, University of Surrey, Guildford, GU2 7XH United Kingdom \\ (Received 17 July 2006; revised manuscript received 29 December 2006; published 11 April 2007)
}

Stable dynamic bound states of dissipative localized structures are found. It is characterized by chaotic oscillations of distance between the localized structures, their phase difference, and the center of mass velocity.

DOI: 10.1103/PhysRevE.75.045601

PACS number(s): 05.45.-a, 42.65.Sf, 42.65.Tg

The complex Ginzburg-Landau equation describes the onset of instability near a Hopf bifurcation in spatially extended systems and, therefore, serves as a universal model for various physical phenomena in hydrodynamics, superconductivity, and optics. In a certain parameter range this equation exhibits a spatially localized solution - a dissipative soliton. In the classical setting with purely cubic nonlinearity, the soliton is unstable, so the next order nonlinear terms should be taken into account in order to describe stable solitons [1]. Indeed, the quintic complex Ginzburg-Landau equation (QCGLE) is widely used in nonlinear optics to describe phenomena related to pulse formation, e.g., mode locking in lasers [2,6], light propagation in nonlinear fibers [3], and transverse pattern formation in nonlinear optical systems [4]. In particular, in mode-locked fiber lasers dissipative solitons appear as short optical pulses propagating along the cavity axis. Well separated from one another, the pulses interact via exponentially decaying tails. Interference between the tails can produce spatial intensity oscillations responsible for the formation of bound states (BS's) of the dissipative solitons (see, e.g., experimental studies in Refs. $[5,6]$ ). Up to now, either stationary and uniformly moving [7-9], or uniformly rotating $[10,11]$ BS's were reported. Here we show that a slight breaking of the phase-shift symmetry yields a huge variety of dynamic BS's, characterized by undamped regular or chaotic oscillations of the solitons positions and phases.

In the course of weak interaction the shape of the solitons is preserved, while their positions and certain internal parameters, such as phases, evolve slowly in time. The GorshkovOstrovsky approach [13,14] allows one to derive a set of finite-dimensional soliton interaction equations (SIE) governing the slow evolution of the soliton parameters. Being independent of the specific details of a model, the form of SIE is determined by the asymptotical behavior of the soliton tails and by the symmetries of the model. When the model admits only translational symmetry, SIE have a gradient structure (see, e.g., Ref. [14]), which implies a trivial dynamics for the weakly interacting solitons. For QCGLE, the additional phase-shift symmetry changes the structure of SIE. Still, the dynamics of the weak two-soliton interaction remains simple and the only attractors are BS's with time-

\footnotetext{
*Electronic address: turaev@math.bgu.ac.il

†Electronic address: vladimir@wias-berlin.de

"Electronic address: S.Zelik@surrey.ac.uk
}

independent distance and phase difference between the solitons $[8,9]$. We show that instead of making the interaction dynamics simpler the phase symmetry breakdown leads to the explosion of the complexity of the two-soliton dynamics (the gradient structure restores only at relatively large values of the symmetry-breaking parameter). Note that the localized in space and chaotic in time regimes which we discover are very different from the earlier known ones, for whom the chaos was a feature of the internal dynamics of a single soliton [12] or was related to a scattering process with unbounded soliton trajectories [15]. In our case, chaos is associated with a strange attractor that forms solely due to the weak soliton interaction.

We consider $1+1$ dimensional QCGLE in the form

$$
\begin{aligned}
\partial_{t} A= & \left(\beta+\frac{i}{2}\right) \partial_{x x} A+A\left[\delta+(\varepsilon+i)|A|^{2}+(\mu+i \nu)|A|^{4}\right] \\
& +\eta \exp (i \Omega t)
\end{aligned}
$$

with complex amplitude $A(x, t)$. Equation (1) is symmetric with respect to spatial translations, and at $\eta=0$ with respect to the phase shifts $A \rightarrow A \exp (i \chi)$. The parameter $\beta>0$ is the diffusion coefficient (the second-order dispersion is scaled to $1 / 2), \delta>0$ describes linear losses; $\varepsilon, \mu$, and $\nu$ define the shape of nonlinearity. An important application concerns Kerr-lens mode-locked lasers [6]. Then, $A$ is a normalized electromagnetic field envelope, and the symmetry breaking term $\eta \exp (i \Omega t)$ corresponds to a weak signal injected into the laser.

Let $A=A_{0}(x) \exp (i \alpha t)$ be a soliton solution at $\eta=0$. Away from the soliton core, $A_{0}(x)$ decays exponentially:

$$
A_{0}(x) \sim p \exp [(-\gamma+i \omega)|x|] \quad \text { as }|x| \rightarrow \infty .
$$

The stability of the soliton is determined by the spectrum of the operator $L_{0}$ obtained by the linearization of the righthand side of Eq. (1) on the soliton at $\eta=0$. Note that $L_{0}$ has two neutral modes, $\Psi(x)=i A_{0}(x)$ and $\Sigma(x)=\partial_{x} A_{0}(x)$, corresponding, respectively, to the phase shift and translational symmetries of the unperturbed QCGLE. It follows that the adjoint operator $L_{0}^{\dagger}$ has two neutral modes as well, $\Psi^{\dagger}(x)$ and $\Sigma^{\dagger}(x)$. We fix their choice by the normalization conditions $\int_{-\infty}^{\infty} \Psi^{\dagger} \Psi d x=1$ and $\int_{-\infty}^{\infty} \Sigma^{\dagger} \Sigma d x=1$. Note that

$$
\Psi^{\dagger}(x) \sim q e^{(-\gamma+i \omega)|x|}, \quad \Sigma^{\dagger}(x) \sim s e^{(-\gamma+i \omega)|x|},
$$

as $|x| \rightarrow \infty$, with certain complex constants $q$ and $s$. 
Up to the leading order in $\exp (-\gamma r)$ (where $r$ is the distance between the solitons), a BS of two weakly interacting solitons has the form

$$
A=\exp (i \alpha t)\left[A_{0}\left(x-x_{1}\right) e^{i \varphi_{1}}+A_{0}\left(x-x_{2}\right) e^{i \varphi_{2}}\right],
$$

where the coordinates $x_{1,2}$ and phases $\varphi_{1,2}$ of the individual solitons are slowly varying functions of time $t$. By plugging this ansatz into Eq. (1) and projecting the resulting equations onto the tangent to the space of functions of type (4), we obtain the following SIE:

$$
\begin{gathered}
\partial_{t} r=a e^{-\gamma r} \sin \left(\omega r+\theta_{1}\right) \cos \varphi, \\
\partial_{t} \varphi=-b e^{-\gamma r} \cos \left(\omega r+\theta_{2}\right) \sin \varphi-c \eta \sin \frac{\varphi}{2} \sin \frac{\Phi}{2}, \\
\partial_{t} \Phi=b e^{-\gamma r} \sin \left(\omega r+\theta_{2}\right) \cos \varphi+c \eta \cos \frac{\varphi}{2} \cos \frac{\Phi}{2}+2 \Delta,
\end{gathered}
$$

$$
V=-a e^{-\gamma r} \cos \left(\omega r+\theta_{1}\right) \sin \varphi
$$

where $\quad r=x_{2}-x_{1}, \quad \varphi=\varphi_{2}-\varphi_{1}, \quad \Phi=\varphi_{1}+\varphi_{2}-2 \zeta, \quad$ and $V=\partial_{t}\left(x_{1}+x_{2}\right) / 2$. The parameter $\Delta=\Omega-\alpha$ is the frequency detuning between the injected field and the single soliton solution. The other parameters are defined by $-a \exp \left(i \theta_{1}\right) / q=b \exp \left(i \theta_{2}\right) / s=4 p[(\gamma-2 \beta \omega)-i(\omega+2 \beta \gamma)]$ and $c \exp (i \zeta)=4 \int_{-\infty}^{\infty} \Psi^{\dagger} d x$, where $p, q, s$ are given by Eqs. (2) and (3).

For more details on the derivation of the SIE, see Ref. [9]. Being obtained formally, using a multiscale method, Eqs. (5)-(8) require a justification. The strongest one is given by the "invariant manifold theorem" of Ref. [16]. It is a general statement which holds for all multisoliton weak interaction processes in a large class of PDE's under the condition of a nonzero diffusion. In our case, for $\beta \neq 0$, the theorem ensures the existence of a closed set of four ODE's ("the true SIE") which give an exact description of the weak interaction of two solitons, valid uniformly on unbounded time intervals. Moreover, it follows from the proof, that the formal scheme employed in the derivation of Eqs. (5)-(8) yields an $o[\exp (-\gamma r)]$ approximation to the true SIE. Note the importance of the nonzero diffusion: for conservative systems, for example, the long-time validity of SIE must be questioned.

For $\eta=0$ the phase-shift symmetry is retained and Eq. (7) for the sum of the soliton phases $\Phi$ decouples from the other equations. Then the dynamics of the soliton interaction is described by the two-dimensional system

$$
\begin{gathered}
\partial_{t} r=a \exp (-\gamma r) \sin \left(\omega r+\theta_{1}\right) \cos \varphi, \\
\partial_{t} \varphi=-b \exp (-\gamma r) \cos \left(\omega r+\theta_{2}\right) \sin \varphi .
\end{gathered}
$$

This system is reversible, i.e., invariant under the transformation $t \rightarrow-t, \varphi \rightarrow \pi-\varphi$. As usual in dimension two, the reversibility implies integrability. The integral is

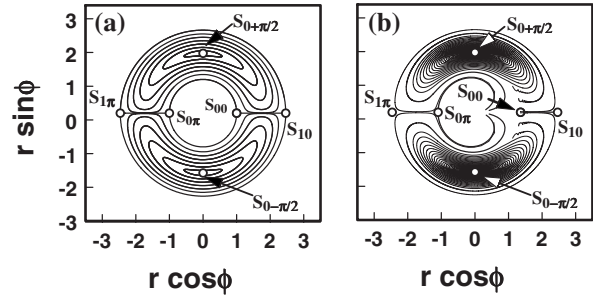

FIG. 1. Phase portraits obtained by numerical solution of (a) the SIE (9) and (b) the QCGLE (1) at $\eta=0$. The parameters in all the figures are $\beta=0.5, \delta=0.02, \varepsilon=1.8, \mu=0.05, \nu=0.05$. This corresponds to $\omega=-2.149, \quad \gamma=5.195, \quad a=0.118, \quad b=7.55 \times 10^{-4}$, $\theta_{1}=6.82 \times 10^{-4}, \theta_{2}=2.25$ in the SIE.

$H=\sin \varphi \exp \left[-b r \sin \left(\theta_{2}-\theta_{1}\right) / a\right]\left|\sin \left(\omega r+\theta_{1}\right)\right|^{b \cos \left(\theta_{2}-\theta_{1}\right) /(a \omega)}$.

The orbits of Eq. (9) comprise the level lines of $H$, so the phase portrait can easily be recovered. Depending on the sign of $\rho=a b \omega \cos \left(\theta_{2}-\theta_{1}\right)$, two types of phase portraits are possible [9]. Here we consider only the case $\rho>0$ when the orbits are closed curves surrounding the neutrally stable equilibria $S_{k \pm \pi / 2}:\left\{\varphi= \pm \pi / 2, \omega r+\theta_{2}=\pi(k+1 / 2)\right\}$ [Fig. 1(a)]. It follows from Eq. (8) that the $\pm \pi / 2$-out-of-phase equilibria correspond to uniformly moving two-soliton states. The saddle equilibria correspond to stationary BS's, in-phase $S_{k 0}:\left\{\varphi=0, \omega r+\theta_{2}=2 \pi k\right\}$ and antiphase $S_{k \pi}:\left\{\varphi=\pi, \omega r+\theta_{2}\right.$ $=\pi(2 k+1)\}$. The separatrices of the saddles divide the phase plane into cells, from which the orbits can never escape [Fig. $1(\mathrm{a})]$.

The higher-order corrections destroy the reversibility, and hence the integrability, of Eqs. (9). Indeed, it is seen from Fig. 1(b) where the results of a direct simulation of Eq. (1) are presented (cf. Ref. [8]), that rather than being closed, orbits slowly spiral towards the weakly stable $\pm \pi / 2$-out-of-phase equilibria. Furthermore, the cells boundaries break: an orbit can flow from cell to cell until it is captured to one of the $\pm \pi / 2$-out-of-phase BS's, or leaves the weak interaction zone.

The boundary of this zone essentially coincides (cf. Ref. [8]) with the inner boundary of the cell shown in Fig. 1(a). In our case the strong interaction does not produce interesting effects: its usual outcome is a collapse of the two-soliton solution into a single soliton, as it is shown in Fig. 2. A detailed discussion of validity of the weak interaction approximation can be found in Ref. [16]. Numerical study of strong interaction of dissipative solitons was performed, e.g., in Ref. [11].

When $\eta \neq 0$, Eq. (7) couples with Eqs. (5) and (6). The dynamics is then determined by the ratios between $\eta, \Delta$, and

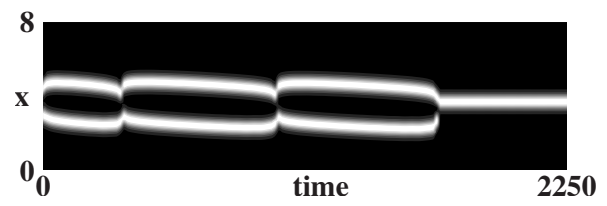

FIG. 2. Merging of two weakly interacting solitons after a transition into the strong interaction zone. The bright spots correspond to higher values of $|A|$. 


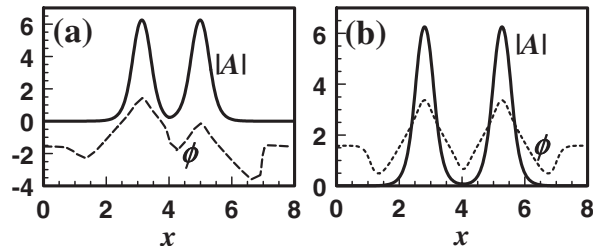

FIG. 3. Stable BS's with the phase difference (a) $\phi=\pi / 2$ and (b) $\phi=0$ between the solitons.

$\exp (-\gamma r)$. Let us show that chaos should be expected when $\Delta \gg \eta$, $\exp (-\gamma r)$. Indeed, as the sum of the soliton phases $\Phi$ rotates with nonzero velocity in this case, $\Phi$ can be taken as a new time variable. Thus, the system (5)-(7) is, effectively, a periodically forced conservative integrable system (9). Such systems do exhibit a chaotic behavior due to the destruction of resonances. So, near every resonance zone, i.e., in the vicinity of those periodic trajectories of Eqs. (9) for which the increment of $\Phi$ during the period is commensurate with $4 \pi$, the soliton interaction dynamics can be chaotic. Since the higher-order corrections introduce a weak dissipation into the SIE, most of the resonances are, in fact, erased. However, as we will see below, the chaotic dynamics produced by the strongest resonances survives.

One more possibility for chaos is the splitting of the cell boundaries. As the phase $\Phi$ rotates, the in-phase and antiphase equilibria of Eqs. (9) become saddle periodic orbits of Eqs. (5)-(7) at $\eta \neq 0$, and their stable and unstable manifolds may intersect. Thus, zones of a "metastable" homoclinic chaos can be formed. This type of behavior is characterized by large oscillations in phase difference $\phi$, i.e., we see chaotic transitions between the cells in the $(r, \phi)$ plane [Fig. 6(g)].

It is noteworthy that the spatial motion of chaotic BS's is, effectively, a random walk at large time scales: as Eq. (8) shows, when the dynamics of $r$ and $\varphi$ is chaotic, the center of mass velocity $V$ is a random function of time (with a certain nonzero decay of correlation time), so the spatial position of the chaotic BS is an integral of a random signal.

Another, nonrotational mechanism of chaos creation in the SIE is related to multiple bifurcations of equilibrium states. As we mentioned, the equilibria of Eqs. (9) correspond, in general, to periodic orbits of Eqs. (5)-(7). However, at moderate values of $\Delta / \eta$, due to a synchronization phenomenon, BS's with stationary $r, \varphi_{1}$, and $\varphi_{2}$ can form (see Fig. 3). The stability domains for the $\pm \pi / 2$-out-of-phase and in-phase equilibria of Eqs. (5)-(7) are shown in Figs. 4(a) and 4(b), respectively. The birth of $\pm \pi / 2$-out-of-phase equilibria is accompanied here by a si-

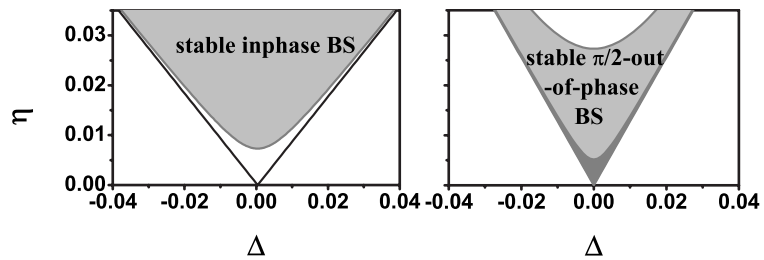

FIG. 4. Stability domains (grey) of the $\pi / 2$-out-of-phase and in-phase BS's on the $(\eta, \Delta)$ parameter plane.
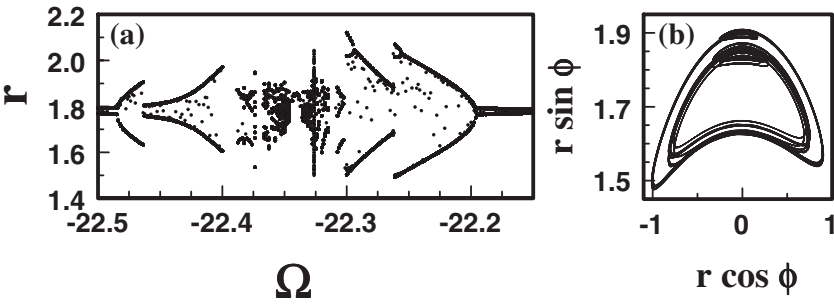

FIG. 5. Simulation results for the QCGLE: (a) bifurcation tree calculated for $\Omega=-22.2$; (b) period three, $\eta=0.065$; (c) chaotic, $\eta=0.06623$; (d) period two, $\eta=0.08$; (e) period six, $\eta=0.083$, soliton BS.

multaneous Andronov-Hopf bifurcation, i.e., in addition to a zero characteristic eigenvalue these equilibria have a pair of pure imaginary eigenvalues. Such double bifurcation is known (see Ref. [17]) to lead to a chaotic behavior via a Shilnikov homoclinic loop. Another multiple instability, also leading to Shilnikov chaos [18], corresponds to a triplet of zero characteristic eigenvalues of the $\pm \pi / 2$-out-of-phase equilibrium at $|\Delta|=c|\eta| / \sqrt{8}=\left(\sqrt{\rho+b^{2} / 4} \pm b / 2\right) \exp \{-\gamma[\pi(k$ $\left.\left.+1 / 2)-\theta_{2}\right] / \omega\right\}$. While higher order corrections to SIE (5)-(7) cause a decrease in the multiplicity of the local bifurcations, the chaos associated with them has to persist nevertheless.

The above analysis is confirmed by the results of simulations of Eq. (1). The two-soliton solutions of Eq. (1) have, at each $t$, two points of maximum of $|A(x, t)|$. We identify them with the soliton positions, $x_{1,2}(t)$; the soliton phases $\varphi_{1,2}$ are then defined as the phases of $A\left(x_{1}(t), t\right)$ and $A\left(x_{2}(t), t\right)$. As one can see from Figs. 5 and 6 , the dynamics of two-soliton states of Eq. (1) can be well represented by the behavior of the variables $r(t)=x_{2}-x_{1}$ and $\varphi(t)=\varphi_{2}-\varphi_{1}$. Figure 5(a) shows the evolution (the "bifurcation tree") for the $\pi / 2$-out-of-phase regime with the change of the injected signal amplitude $\eta$. Away from the synchronization range, the regime undergoes a number of period-doubling and periodtripling bifurcations (strong 1:2 and 1:3 resonances) leading, in particular, to chaotic behavior [see Fig. 5(b)]. Different other dynamical regimes are shown in Fig. 6 where the phase portraits in the upper row are obtained by the numerical so-
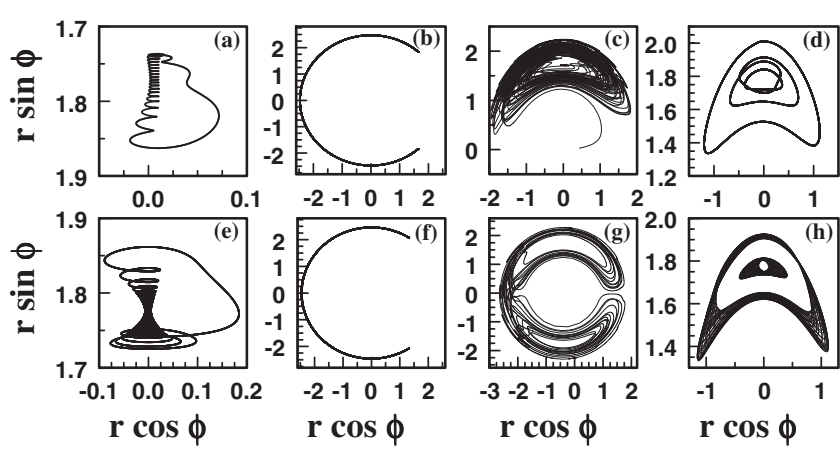

FIG. 6. Phase portraits obtained by solving numerically the QCGLE (a)-(d) and SIE (e)-(h). (a) $\eta=0.02, \Omega=-22.3465$; (b) $\eta=0.4, \quad \Omega=-22.2 ;$ (c) $\eta=0.06, \Omega=-22.2 ;$ (d) $\eta=0.02$, $\Omega=-22.25$; (e) $\eta=0.02, \Delta=-0.0164$; (f) $\eta=0.02, \Delta=0.023$; (g) $\eta=0.02, \Delta=0.11$; (h) $\eta=0.11, \Delta=0.41$. 
lution of Eq. (1), while those in the lower row correspond to Eqs.(5)-(7). Figures 6(a) and 6(e) illustrate a desynchronization transition from the stationary $\pi / 2$-out-of-phase BS to a stable limit cycle via a homoclinic bifurcation (cf. Ref. [19]). A stable limit cycle born from a homoclinic loop to a saddle antiphase state is shown in Figs. 6(b) and 6(f). Figure 6(c) shows a metastable chaotic BS which corresponds to a stable chaotic regime of Eqs. (5)-(7) [see Fig. 6(g)]. Finally, Fig. 6(d) illustrates multistability between different time-periodic BS's of Eq. (1). The corresponding quasiperiodic solutions of Eqs. (5)-(7) are shown in Fig. 6(h). The comparison of the phase portraits in the upper and lower rows in Fig. 6 reveals a substantial similarity between the solutions of Eq. (1) and those of Eqs. (5)-(7). There is no one-to-one correspondence, however, because in Eqs. (5)-(7) we have neglected second- and higher-order terms in $\exp (-\gamma r)$ that are responsible for a weak dissipation. Though these terms are small, they are not negligible in the first two elementary cells of the phase space that are depicted in Fig. 1(a). In the next cells that correspond to larger soliton separations one should expect a similar behavior, though with much weaker dissipation effects.

As we see, the weak interaction of two dissipative solitons can produce a very rich dynamics. The mechanism of a cre- ation of dynamic soliton BS's is related to the breakdown of the phase-shift symmetry and has a universal, modelindependent nature. Therefore, it should be typical for every spatially extended system which undergoes a Hopf bifurcation. In particular, in mode-locked lasers violation of the phase-shift symmetry can be easily achieved by an injection of an external signal. An experimental technique allowing one to measure the parameters of interacting solitons with a good precision is described in Refs. [5,6]. This technique, based on autocorrelation and spectral analysis of laser output radiation, seems to be quite capable of detecting the dynamic soliton BS's and distinguishing them from the static ones.

The fact that the two-soliton state in the QCGLE is a weakly damped nonlinear oscillator can be used to analyze the dynamics of soliton BS's in other situations. Thus, a system of four interacting solitons can be viewed as a pair of coupled, weakly damped oscillators, i.e., is able to demonstrate a rich dynamical behavior even without the phase-shift symmetry breaking. Similar effects can be expected in the case of rotating soliton BS's in two space dimensions.

This work was supported by Grant ISF No. 926/04 and the joint Russian-Israeli project "Global bifurcations and complex orbit behavior."
[1] O. Thual and S. Fauve, J. Phys. (France) 49, 1829 (1988).

[2] J. D. Moores, Opt. Commun. 96, 65 (1993).

[3] N. N. Akhmediev and A. Ankiewicz, Solitons. Nonlinear Pulses and Beams (Chapman and Hall, London, 1997).

[4] P. Mandel and M. Tlidi, J. Opt. B: Quantum Semiclassical Opt. 6, R60 (2004).

[5] D. Y. Tang, W. S. Man, H. Y. Tam, and P. D. Drummond, Phys. Rev. A 64, 033814 (2001); D. Y. Tang, B. Zhao, D. Y. Shen, C. Lu, W. S. Man, and H. Y. Tam, ibid. 68, 013816 (2003); P. Grelu et al., Opt. Lett. 27, 966 (2002); Opt. Express 11, 2238 (2003).

[6] P. Grelu and N. Akhmediev, Opt. Express 12, 3184 (2004).

[7] H. R. Brand and R. J. Deissler, Phys. Rev. Lett. 63, 2801 (1989); B. A. Malomed, Phys. Rev. A 44, 6954 (1991); V. V. Afanasjev and N. Akhmediev, Phys. Rev. E 53, 6471 (1996).

[8] N. N. Akhmediev, A. Ankiewicz, and J. M. Soto-Crespo, Phys. Rev. Lett. 79, 4047 (1997).

[9] A. G. Vladimirov, G. V. Khodova, and N. N. Rosanov, Phys. Rev. E 63, 056607 (2001).

[10] D. V Skryabin and A. G. Vladimirov, Phys. Rev. Lett. 89, 044101 (2002).
[11] N. N. Rosanov, S. V. Fedorov, and A. N. Shatsev, Phys. Rev. Lett. 95, 053903 (2005).

[12] J. M. Soto-Crespo, N. Akhmediev, and A. Ankiewicz, Phys. Rev. Lett. 85, 2937 (2000).

[13] K. A. Gorshkov, L. A. Ostrovsky, and V. V. Papko, Sov. Phys. JETP 44, 306 (1976); K. A. Gorshkov and L. A. Ostrovsky, Physica D 3, 428 (1981).

[14] I. S. Aranson et al., Physica D 43, 435 (1990).

[15] K. A. Gorshkov, A. S. Lomov, and M. I. Rabinovich, Nonlinearity 5, 1343 (1992).

[16] B. Sandstede, in Handbook of Dynamical Systems II, edited by B. Fiedler (Elsevier, Amsterdam, 2002); S. Zelik and A. Mielke, Memoirs of AMS (in press).

[17] J. Guckenheimer and P. Holmes, Nonlinear Oscillations, Dynamical Systems, and Bifurcations of Vector Fields (Springer, Berlin, 1983).

[18] A. Arneodo et al., Physica D 14, 327 (1985); S. Ibáñez and J. A. Rodríguez, J. Differ. Equations 208, 147 (2005).

[19] N. K. Gavrilov and A. L. Shilnikov, Am. Math. Soc. Transl. 200, 165 (2000); B. Krauskopf and S. Wieczorek, Physica D 173, 114 (2002). 